\title{
PLATERÍA EN LA COLONIA: LA HISTORIA DE DOS ATRILES
}

\author{
Colonial silversmithing: the story of two lecterns.
}

\author{
Andrea Lorena Guerrero Jiménez \\ jilguerrero@poligran.edu.co \\ Valentina Ovalle Camargo \\ vaovalle1@poligran.edu.co \\ Nadia Catalina Flórez Soaita \\ naflorez3@poligran.edu.co \\ Sunny Johana Romero Sánchez \\ sjromeros@poligran.edu.co \\ Camila Andrea Guzman Franco \\ caguzman71@poligran.edu.co \\ Politécnico Grancolombiano \\ Diseño Industrial \\ Colombia
}

\section{RESUMEN}

Este texto presenta el estudio de dos piezas de platería neogranadina, cuya realización está datada en la segunda mitad del siglo XVII, en un contexto social multifacético influenciado por manos españolas e indígenas. Se expone cómo el análisis de estos artefactos, desde el punto de vista del diseño industrial, permite ver aspectos que otras disciplinas estudian superficialmente, como la forma y la función de un objeto. El objetivo es entender el contexto histórico de una sociedad a través del uso de sus objetos, su simbología y las dinámicas de su manufactura. Esta investigación se desarrolló en alianza con el Museo Colonial de Bogotá, el cual posibilitó el acceso directo a la pareja de atriles, lo que permitió la verificación de huellas técnicas para establecer su proceso de elaboración; a pesar de la falta de marcajes coloniales reglamentarios en las piezas, se pudo indagar sobre el contexto histórico de la Nueva Granada y las técnicas plateras que se aplicaron en estos artefactos, tanto en las fuentes secundarias de investigadores expertos en el tema, como en la contrastación con la información técnica directa suministrada por un maestro de platería actual. Se concluye que el papel de los plateros en la Nueva Granada era de vital importancia para los propósitos que tenía la Corona española de expandir la religión católica por todo el Imperio, pues por medio de la realización de objetos de culto en metales preciosos, se lograba representar la devoción y a su vez capturar la atención de los feligreses, debido al alto nivel de detalle decorativo influenciado por el movimiento Barroco.

\section{PALABRAS CLAVE:}

Platería neogranadina, Nueva Granada, técnicas de platería, historia colonial

Cite este artículo como: Guerrero, A., Ovalle, V., Flórez, N., Romero, S., \& Guzmán, C. (2020). Platería en la Colonia: la historia de dos atriles. 


\section{INTRODUCCIÓN}

Este proyecto surge en el marco del Semillero de Investigación Diseño, Artefacto y Sociedad en conjunto con el Museo Colonial, alianza por la cual se tuvo acceso una pareja de atriles de plata de la segunda mitad del siglo XVII, elaborados presumiblemente en la Nueva Granada. El objetivo de la investigación fue entender el origen, el contexto y, de manera específica para este caso, establecer las técnicas bajo las cuales se elaboraron estos dos artefactos; todo ello desde el punto del diseño industrial, que entiende los objetos desde su forma y función, partiendo de la premisa de que estos dos componentes están estrechamente ligados a la relación que tienen las personas con los objetos. El proceso y los resultados expuestos en el presente artículo hacen parte de un proyecto de investigación más amplio, cuyo objetivo fue la caracterización integral de este par de piezas coloniales.

Entender un momento específico de nuestra historia (período colonial), por medio del análisis de un artefacto resultó ser un reto interesante, pues se descubre que los elementos materiales tienen muchas historias que contar. Con este enfoque se logra preservar la memoria histórica, ya que el mismo estudio de nuestro pasado nos comprometió a "revivirlo" y a entender la importancia del oficio de la platería y el papel que cumplía en el desarrollo de las actividades religiosas de esta época específica.

Durante el proceso de investigación se indagó la presencia de las marcas reglamentarias en las piezas (quintado, ensaye, ciudad, artífice) (Esteras, 1992), pero la pareja de atriles no presenta ninguna de las cuatro marcas, lo que dificulta establecer el origen específico y el autor; sin embargo, el estudio directo de los objetos nos permitió delimitar unas fechas tentativas y hacer aproximaciones a partir de las técnicas utilizadas, su iconografía y el propósito final de los artefactos. Con la intención de hacer un estudio detallado de esta pareja de atriles pertenecientes a la colección de Platería del Museo Colonial, se parte de la observación de las huellas técnicas de las piezas, y su contrastación con las fuentes secundarias y primarias (asesoría directa con un maestro platero experto).

\section{MÉTODO}

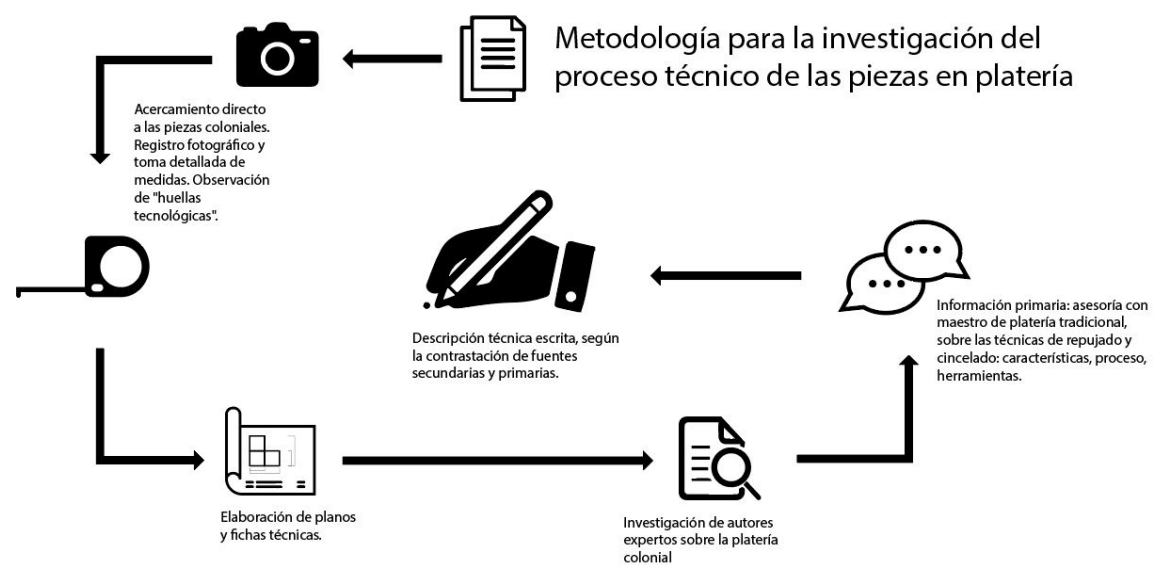

Previo al levantamiento de las fichas técnicas de las piezas se realizó un balance bibliográfico general sobre la platería neogranadina, en el cual se incluyeron las investigaciones realizadas por Marta Fajardo en su documento Oribes y plateros en la Nueva Granada (2008), Los faroles procesionales, un cuaderno de estudio, publicado por el Museo Colonial (2003), Plata y oro para la fe - cultura tridentina e iconología cristiana en la platería neogranadina virreinal (2018) y la Tesina de maestría De la materia al artefacto: relaciones sociales, productivas y simbólicas en la platería neogranadina virreinal (2009), de Lorena Guerrero Jiménez, docente 
líder del Semillero. Igualmente, fue una referencia importante la más reciente publicación del Museo Colonial, el Volumen IV de su Catálogo, que incluye cuatro capítulos de expertos en la temática (2019). Siguiendo las referencias metodológicas encontradas en estos textos, se propone un estudio integral y detallado de la pareja de atriles, desde los aspectos de uso, producción e iconología, a la luz de las condiciones del contexto. Para el caso de este artículo, vamos a detallar en los avances de la investigación sobre las técnicas de producción con las que fueron elaborados.

En primer lugar, se hizo el acercamiento a las piezas de platería a partir de lo cual se realizó el levantamiento de cada una de las fichas técnicas donde se especifican las medidas, los materiales y técnicas utilizadas, y a su vez el estado actual de las piezas. Con las medidas recopiladas se dibujaron los planos técnicos de las dos piezas de platería y sus respectivas fichas técnicas.

Posteriormente, se procedió a profundizar en la investigación historiográfica, recurriendo a autores que previamente han escrito sobre la platería en la Nueva Granada; así mismo, el Museo Colonial nos facilitó los Cuadernos de Estudio que han publicado con respecto a este tema.

Como último avance de este proceso de investigación en curso sobre la descripción técnico-productiva de las piezas, se realizó un contacto con un maestro platero, quien nos brindó información muy valiosa sobre las técnicas tradicionales aplicadas en los atriles, las herramientas y procesos al detalle.
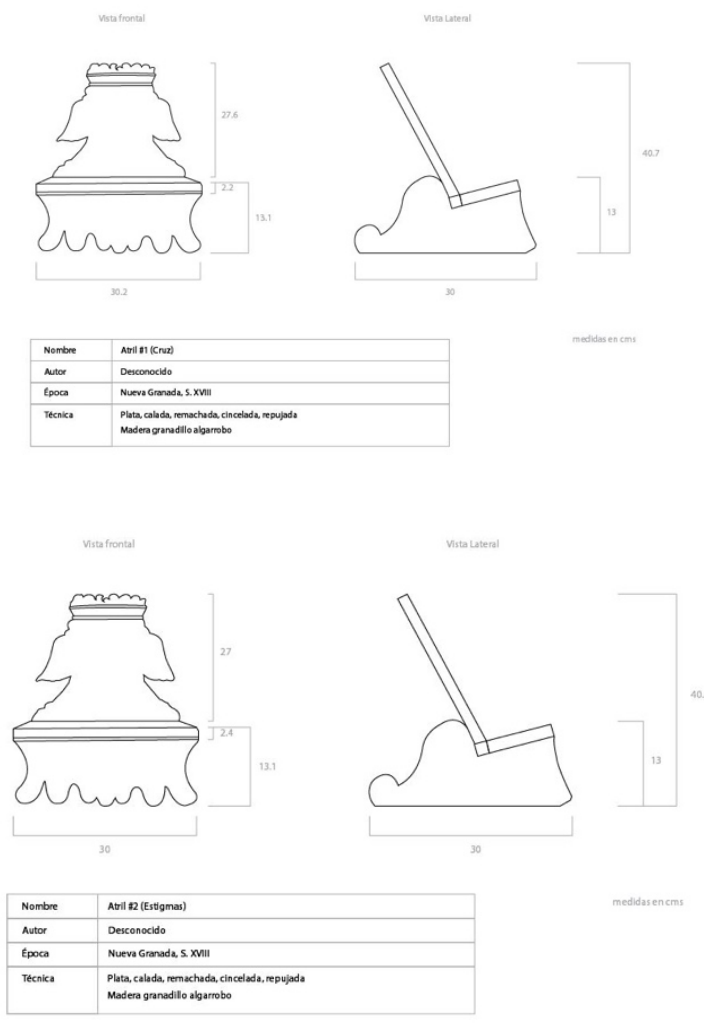

Imagen 2. Imagen de Planos y fichas técnicas Atriles de Águila Bicéfala-Museo Colonial Elaborados por: Nadia Flórez 


\section{RESULTADOS}

A partir del estudio de objetos históricos, ya sean de uso o de culto, se pueden entender rasgos de una sociedad como su contexto social, cultural, político, económico y religioso; de acuerdo con Guerrero (2009), los objetos son traductores de la historia. En este orden de ideas, se plantea que la indagación sobre el origen de dos atriles en plata nos puede dar luces sobre algunos de los comportamientos sociales y culturales de la Nueva Granada en el período colonial.

Los hallazgos de esta investigación permiten documentar que estas piezas de platería pertenecen al siglo XVII (datación Museo Colonial), un contexto de convergencia de muchas ideologías, razas y religiones que se definieron a sí mismas o por medio del choque y la convivencia, dando como resultado una combinación diversa que se ve representada en las manifestaciones materiales y simbólicas de sus artesanos.

La mayoría de estas piezas eran para propósitos y usos eclesiásticos, pues eran la iglesia y las comunidades religiosas quienes más encargaban su elaboración a los maestros plateros (Guerrero, 2009). Sin embargo, estos encargos también los hacían civiles, miembros de familias pudientes, que demostraban su devoción por medio de la ostentación, pues entre más lujoso fuese el artefacto, expresaba mayor veneración. Igualmente, con la llegada de familias españolas plateras, llega nuevo conocimiento, técnicas e instrumentos para la elaboración de artefactos en metales preciosos, que ya tenía un desarrollo importante por parte de las comunidades precolombinas.

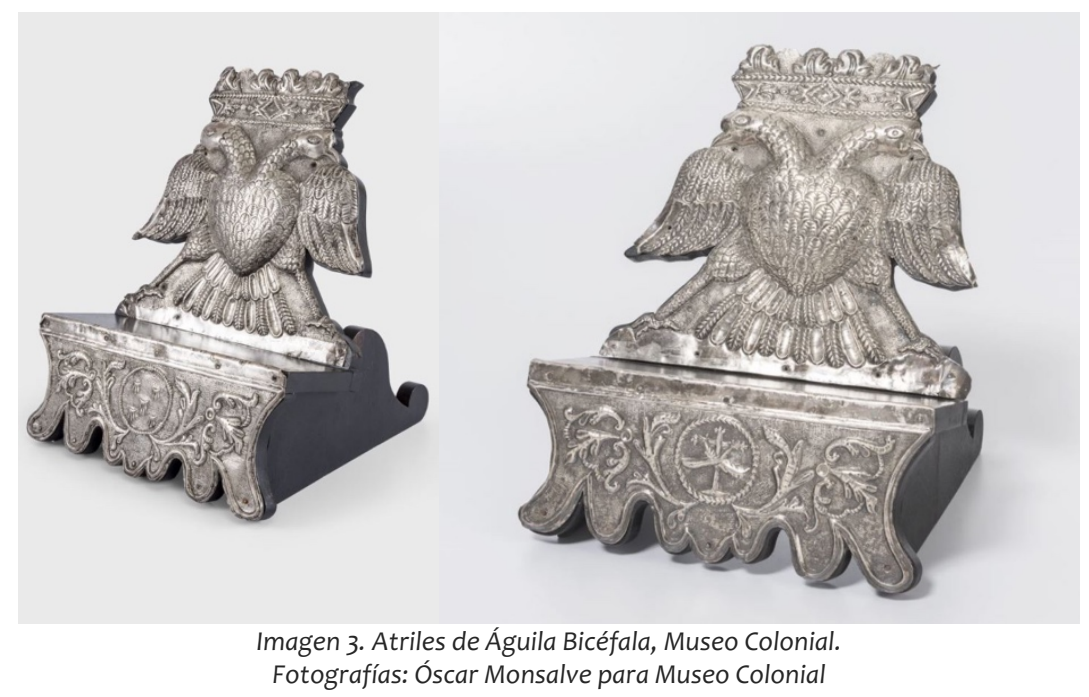

En el análisis técnico de los atriles de águila bicéfala se identificaron cuatro técnicas propias de la platería desde el siglo XVII en adelante, siendo las dos primeras ornamentales, y las otras dos constructivas.

Antes de la aplicación de las técnicas como tal, debía hacerse un proceso de preparación de la plata, para que posteriormente pudiera ser trabajada desde una lámina; lo primero que se hace es fundir el metal para compactarlo cuando se hacen aleaciones o cuando el metal utilizado es de una pieza ya existente. Luego de tener un lingote fundido se golpeaba con un martillo para ir formando la lámina, proceso que se realizaba sobre un yunque o bigornia. Para este laminado era indispensable que el metal se calentara previamente (recocido), para conservar su maleabilidad. En la medida que va siendo martillado, el metal vuelve a adquirir temple, por lo que debía hacerse el proceso de recocido varias veces durante la obtención de la lámina, condición que continúa en las técnicas actuales. 
Luego del proceso de manufactura de la lámina, se procedía a aplicar las técnicas ornamentales y de construcción.

En primer lugar, se encontró el repujado, técnica utilizada en la mayor parte de los dos atriles, como en las hojas de acanto de la parte superior, el corazón y las cabezas del águila bicéfala, las alas, plumas y patas del águila; y en la parte inferior que es la que muestra una diferencia marcada en las dos piezas; por un lado, tenemos el cordón de hojas, el escudo franciscano, las volutas de acanto y el borde de la pieza en el primer atril, y por otro lado, el cordón con nudos, los cinco estigmas, las volutas de acanto y los bordes de la pieza en el segundo atril.

El repujado consiste en realizar figuras por el reverso de la pieza, dando suaves golpes a la plata con embutidores y cinceles de diferentes formas y tamaños, sobre una pasta especial de cincelar que amortigua los golpes del martillo y evita que la plata se rompa, dando como resultado un relieve positivo en la parte frontal de la pieza.

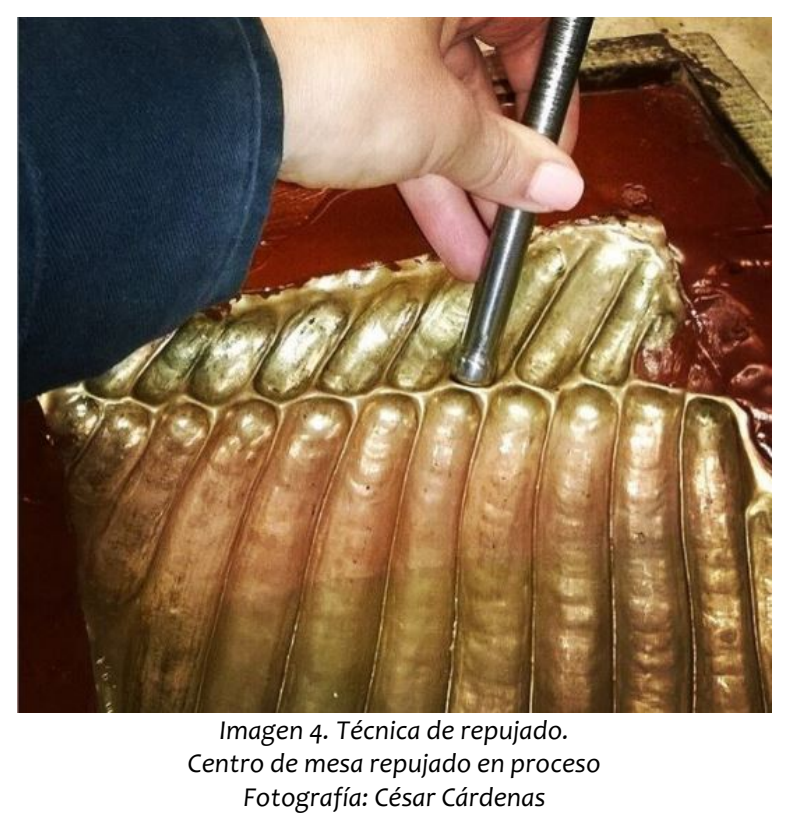

En segundo lugar, se evidencia claramente el cincelado; al igual que el repujado, en esta técnica son utilizados los cinceles, pero por el anverso de la lámina, obteniendo como resultado un relieve negativo; esta técnica es utilizada para los pequeños detalles y texturas sobre la forma repujada.

En el caso de los atriles, esta técnica se ve claramente en los detalles, que son los que definen las figuras iconográficas; se evidencia en las hojas de acanto cada una de las plumas, los ojos, el pico y las patas del águila bicéfala, y en la parte inferior, los detalles de cada una de las figuras allí presentes, además de hacer un cincelado de puntillismo en la superficie de los dos atriles. 


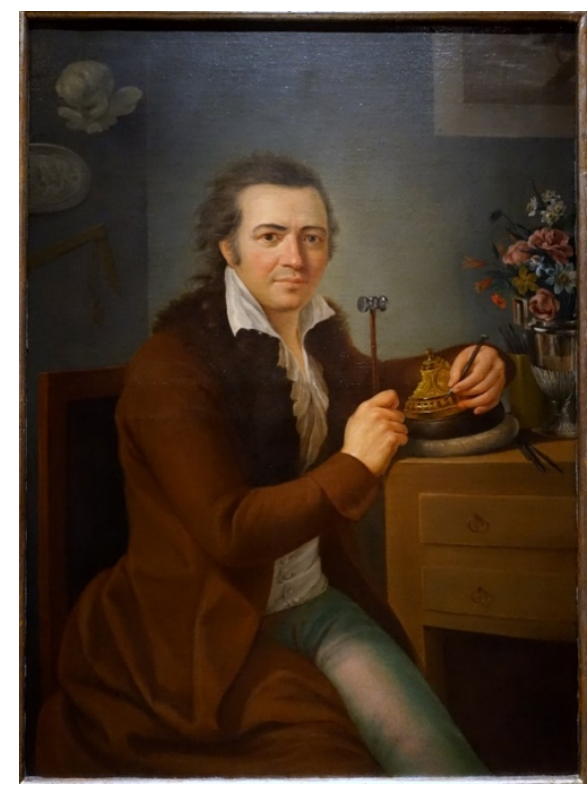

Imagen 5. Platero José María Rodallega Óleo sobre lienzo, Rafael Ximeno y Planes, 1795 Colección Siegel, México

Tras el proceso de técnicas ornamentales en las dos piezas, vienen las técnicas constructivas; por un lado, está el calado, que se hacía con cinceles de corte y que permitía el contorneado de la pieza, para que posteriormente sea unida a la base de madera por medio de remaches.

Como se puede evidenciar, en estas dos piezas de platería las técnicas más importantes son el repujado y cincelado; en esta pareja de atriles el cincelado en particular no presenta un detalle de alta calidad, probablemente debido a que no se usaron herramientas muy especializadas en su elaboración; aun así, cumplen con el objetivo de plasmar correctamente lo que quieren transmitir, puesto que la iconografía está llena de significados devocionales, aspectos que se detallarán en otras publicaciones y productos derivados de esta investigación.

Como resultados preliminares de esta investigación, se entregó al Museo Colonial toda la información pertinente para la realización de una infografía sobre la iconografía de esta pareja de atriles, con ilustraciones detalladas de sus motivos, que al corriente está expuesta en la Exposición temporal Lo sagrado y lo humano: Platería en el Nuevo Reino de Granada, y que estará abierta al público hasta mediados de 2021. Igualmente, se ha finalizado el texto definitivo para la publicación de un Cuaderno de Estudio dedicado a esta interesante pareja de Atriles, y se tiene proyectado la realización de material audiovisual y pedagógico con motivo de la contrastación de las técnicas del platero en el período colonial y en la actualidad.

\section{DISCUSIÓN Y CONCLUSIÓN}

La mayoría de las investigaciones que se realizan a piezas históricas son desde el punto de vista de historiadores, antropólogos o arqueólogos. Este trabajo ofrece el punto de vista del diseño industrial, que entiende a los artefactos como actores de cambio y representantes de culturas, más que como simplemente objetos de uso. Nuestro análisis desde la forma y la función dan una mirada innovadora la manera de analizar el trabajo de la platería, pues se entiende de manera personal y casi íntima la manera en que funcionan los talleres y los materiales. 
Una de las problemáticas a la hora de realizar la indagación histórica de estas dos piezas de platería es la falta de marcajes en las piezas. Por ley, las piezas de platería deberían tener cuatro marcas para su correcta identificación. Se entendía por marcajes los cuatro sellos que debían tener los artefactos elaborados por los plateros: la marca del platero, la marca de ciudad, la marca de ensayador y la marca de quintado. El propósito de estos sellos era asegurar el pago de impuestos, el cumplimiento de las leyes de aleaciones (la pureza de la aleación del metal), y controlar la circulación de las piezas entre provincias y Reales Audiencias. Sin estos sellos resulta difícil identificar el autor de las piezas, pero no limita la capacidad de indagar sobre el funcionamiento y dinámicas de los talleres de platería, que contaban con un gran prestigio al trabajar piezas en su mayoría para la devoción y el culto religioso.

Es imposible devolverse en el tiempo, tan solo se nos permite soñar con que estos marcajes se hubieran realizado, pues de estar presentes se podría tener datos más precisos sobre las piezas. En cuanto a lo que concierne al presente de este oficio, se sabe que investigar sobre su historia permite preservar la memoria histórica de la labor de cientos de artesanos que cuentan su historia anónima por medio de metales preciosos. Recordar técnicas ancestrales ayuda a mejorar las actuales, pues nos puede dar alternativas a procesos que, aunque más eficientes, resultaron ser más contaminantes y perjudiciales para el ambiente.

En este orden de ideas es posible concluir que el estudio de la cultura material desde el enfoque del diseño industrial puede tener grandes resultados: por un lado, como proceso de formación en la investigación, el acercamiento a piezas patrimoniales es un valioso aprendizaje, pues promueve el pensamiento relacional entre contexto y artefactos; la comprensión de las permanencias y transformaciones de las técnicas productivas de un material contribuye a reconocer el legado de patrimonio inmaterial que sigue vivo en los artesanos contemporáneos para darle valor, promoción y reconocimiento; por último, se comprende que con la generación de nuevo conocimiento surge el compromiso de su transferencia a la sociedad, ya sea a un público amplio a través de las entidades museales, o a un grupo más específico como los joyeros y orfebres actuales, cuya permanencia geográfica en los centros de producción se nos revela como un arte de resistir ante los avatares de la historia y la modernización.

\section{REFERENCIAS BIBLIOGRÁFICAS}

Contreras, V., \& Ramírez, F. (2003). Los faroles procesionales. Bogotá: Museo de Arte Colonial, Ministerio de Cultura.

Esteras, C. (1992). Marcas de Platería Hispanoamericana. Madrid: Tuero.

Fajardo, R. (2008). Oribes y plateros en la Nueva Granada. León: Universidad de León, Secretariado de Publicaciones.

Guerrero, L. (2009). De la materia al artefacto: relaciones sociales, productivas y simbólicas en la platería neogranadina virreinal. Bogotá: Tesina de Maestría en Historia, Pontificia Universidad Javeriana.

Guerrero, L. (2018). Plata y oro para la fe - cultura tridentina e iconología cristiana en la platería neogranadina virreinal. Bogotá: H-ART: revista de historia, teoría y crítica de arte, (3), 49-78.

Guerrero, L. (2019). Las técnicas del platero: saberes y prácticas mecánicas como soporte gremial en la platería neogranadina. Ponencia presentada en el VII Congrego Internacional La Plata en Hispanoamérica. 16-22 de septiembre de 2019.

Ministerio de Cultura (2019). Catálogo Museo Colonial, Volumen IV Platería. Bogotá: Ministerio de Cultura. 